Themenzyklus der Kriegsberichterstattung: Ein Phasenmodell

by P. Miltner \& A. Waldherr

To cite this article:

Miltner, Peter \& Waldherr, Annie (2013): Themenzyklus der Kriegsberichterstattung: Ein

Phasenmodell. Publizistik, 58, 267-287.

Published in:

Publizistik (Link to article)

Copyright:

The Authors, 2013

DOI:

10.1007/s11616-013-0180-2 


\title{
Themenzyklus der Kriegsberichterstattung - Ein Phasenmodell
}

\author{
Peter Miltner • Annie Waldherr
}

\section{Zusammenfassung:}

In diesem Beitrag analysieren wir Kriegsberichterstattung aus einer Längsschnittperspektive. Das in der Kommunikationswissenschaft häufig diskutierte Themenzyklusmodell übertragen wir auf medial beachtete Kriege. Dabei differenzieren wir nach der Dauer und Vorhersehbarkeit der Kriegsereignisse verschiedene Typen von Themenverläufen mit unterschiedlichen Phasen. Dabei nehmen wir an, dass sich die Berichterstattung von Phase zu Phase nicht nur quantitativ, sondern auch in ihren inhaltlichen Merkmalen unterscheidet. Anhand der Berichterstattung über den Libanonkrieg 2006 in den Tageszeitungen Süddeutsche Zeitung und Frankfurter Allgemeine Zeitung wird das vorgeschlagene Modell empirisch exploriert. Die Analyse zeigt, dass die Berichterstattung in diesem Fall in vier verschiedene Phasen eingeteilt werden kann und vor allem ereignisgetrieben ist. Die größte Stimmenvielfalt bezüglich der Sprecher, die zu Wort kommen, herrscht in der Hochphase der Berichterstattung, in der insbesondere Medienund Kriegsakteure deutlich mehr Raum in der Berichterstattung einnehmen als in den anderen Phasen.

Schlüsselwörter: Kriegsberichterstattung - Themenzyklus - Phasenmodell - Längsschnittbetrachtung

P. Miltner, Dipl. rer. com. $(\bowtie) \cdot$ Dr. A. Waldherr Institut für Publizistik- und Kommunikationswissenschaft, Freie Universität Berlin Garystr. 55, 14195 Berlin, Deutschland E-Mail: peter.miltner@fu-berlin.de

Dr. A. Waldherr

E-Mail: annie.waldherr@fu-berlin.de 


\begin{abstract}
:
In this article, we analyse war coverage from a longitudinal perspective. In doing so, we refer to the classical issue cycle model, which has been discussed frequently in communication science. We adapt the model to war coverage and - according to the duration and the predictability of war events - we distinguish different types of news cycles. We assume that different stages of news coverage differ not only quantitatively, but also qualitatively with regards to the cited sources. The coverage on the war in Lebanon (2006) in the newspapers Süddeutsche Zeitung and Frankfurter Allgemeine Zeitung serves as a case study for the empirical exploration of the suggested model. The coverage can be divided into four different phases and the results show that it was mainly event-driven. In the peak phase of coverage, increased citations of actors participating in the conflict and of other media actors are observed, as well as generally the most diverse range of voices to be heard in the coverage.
\end{abstract}

Keywords: war coverage - issue cycle - phase model - longitudinal analysis 


\section{Themenzyklus der Kriegsberichterstattung - Ein Phasenmodell}

Kriege wurden von den Medien seit jeher stark beachtet und stellen ein wichtiges und vorrangiges Berichterstattungsthema dar (vgl. Löffelholz 2004; Wilke 2005). Auch als Forschungsgegenstand der Kommunikationswissenschaft haben Kriege und ihre mediale Berichterstattung große Relevanz erlangt (für einen Überblick vgl. u. a. Eilders und Hagen 2005; Löffelholz 2004). Inhaltlich konzentriert sich die Forschung u. a. auf Tendenzen (vgl. z. B. Bennett 1990), Frames (vgl. Entman 2003; Iyengar und Simon 1994) und Medienwirkungen der Berichterstattung - wie etwa den Rally-Effekt (vgl. Bytzek 2005). Inhaltliche Muster der Berichterstattung wie Bewertungen oder Frames werden dabei jedoch vorwiegend im Querschnitt betrachtet. Längsschnittstudien sind bisher noch die Ausnahme (vgl. z. B. Fröhlich 2010; Fröhlich et al. 2007), gewinnen aber zunehmend das Interesse der Forscher. Auffallend ist, dass viele Studien eine dynamische Betrachtung der Berichterstattung gleichsam nur als Nebenprodukt einer eigentlichen Querschnittuntersuchung liefern (vgl. z. B. Bytzek 2007; Hils 2007; Krüger 2003). Auch schlagen manche Autoren zwar konkrete Phaseneinteilungen der Kriegsberichterstattung vor (vgl. z. B. Löffelholz 2004; Savarese 1993), eine Integration dieser Betrachtungen mit allgemeinen kommunikationswissenschaftlichen Themenzyklusmodellen steht bisher aber noch aus.

Die vorliegende Studie nimmt deshalb explizit die Zeitperspektive der Kriegsberichterstattung in den Blick. Unter Kriegsberichterstattung wird dabei mit Staiger (2004, S. 152) die „mediale Thematisierung von Aspekten, die Bezug zu einem Krieg herstellen“ verstanden. Hierzu zählt nicht nur die Berichterstattung während eines Krieges, ${ }^{1}$ sondern auch die Vor- und Nachkriegsberichterstattung. Ziel der Studie ist es, basierend auf

\footnotetext{
${ }^{1}$ Für die Kriegsdefinition soll hier das Verständnis der Arbeitsgemeinschaft Kriegsursachenforschung gelten, die unter Krieg einen gewaltsamen Massenkonflikt zwischen mind. zwei bewaffneten Streitkräften versteht, der ein Mindestmaß an Kontinuität und zentral gelenkter Organisation aufweisen muss (Schreiber 2008: 8). Die Definition macht es möglich, Anfang und Ende von Kriegen zu identifizieren und somit auch zwischen Vor- und Nachkriegsberichterstattung zu unterscheiden.
} 
den vorhandenen theoretischen und empirischen Arbeiten ein Phasenmodell für die Einteilung und Untersuchung von Kriegsberichterstattung zu entwickeln, das sich am klassischen Themenzyklusmodell nach Luhmann (1970) bzw. Downs (1972) orientiert. Somit soll die dynamische Betrachtung von Kriegsberichterstattung vorangetrieben und unter theoretischen Gesichtspunkten diskutiert werden.

Die dynamische Betrachtung von Berichterstattung im Sinne eines Lebenszyklus von Themen hat in der Kommunikationswissenschaft - angefangen mit den Arbeiten von Luhmann (1970) und Downs (1972) - eine lange Tradition. Studien dieser Art erlauben einen differenzierten Blick auf die mediale Berichterstattung im Zeitverlauf. Sie identifizieren kritische Schwellen und Punkte, bei denen ein Thema zum Durchbruch kommt (vgl. Berens 2001; Brosius und Eps 1993; Kepplinger und Habermeier 1995; Weßler 1999) und zeigen zum Beispiel, dass verschiedene Akteure in unterschiedlichen Phasen des Themenzyklus unterschiedliche Handlungsspielräume haben (vgl. Wolfsfeld und Sheafer 2006; Waldherr 2012).

Auch für die Kriegsberichterstattung hat diese Forschungsperspektive eine erhebliche Relevanz. Ein klassischer Konflikt um die Thematisierung von Kriegen liegt z. B. zwischen dem Informationsinteresse der Öffentlichkeit und den Interessen der kriegsführenden Akteure. Welche Informationen werden offiziell freigegeben, welche durch die Militärs geheim gehalten? Wer erhält wann und wie Zugang zum Kriegsgeschehen und die Möglichkeit, sich dazu zu äußern; und wann haben regierungs- und kriegskritische Akteure die größten Chancen mit ihren Ansichten öffentliches Gehör zu finden? Hier ist davon auszugehen, dass Regierungsparteien und Militärs die öffentliche Agenda in unterschiedlichen Phasen des Themenzyklus unterschiedlich stark beeinflussen können und demnach Geheimhaltungs- bzw. Aufdeckungsstrategien der Akteure unterschiedliche Chancen auf Erfolg haben. 
In der nachfolgenden Untersuchung soll geprüft werden, inwieweit sich das klassische Themenzyklusmodell eignet, um die typische Entwicklung von Kriegsberichterstattung im Zeitverlauf systematisch zu erforschen. Dabei soll auch geklärt werden, in welchen Punkten das klassische Modell abgeändert oder erweitert werden muss, um den Besonderheiten von Kriegen als medial beachtete und berichtete Ereignisse gerecht zu werden. In Abschnitt 1 werden zunächst verschiedene Einflussfaktoren von Kriegsberichterstattung systematisiert, worauf in Abschnitt 2 die dynamische Perspektive eingeführt wird. Hier werden zum einen Phasenmodelle der Kriegsberichterstattung, zum anderen die Grundzüge der kommunikationswissenschaftlichen Themenzyklusforschung vorgestellt. Die Erkenntnisse beider Ansätze werden in Abschnitt 3 in ein ganzheitliches Phasenmodell integriert, welches den quantitativen Verlauf der Kriegsberichterstattung vor, während und nach einem Krieg modelliert. Nach dieser theoretischen Systematisierung folgt in Abschnitt 4 eine empirische Exploration anhand der Berichterstattung zweier deutscher Qualitätszeitungen zum Libanonkrieg 2006.

\section{Einflussfaktoren der Kriegsberichterstattung}

Um die Dynamik der Kriegsberichterstattung im Zeitverlauf zu untersuchen, ist es zunächst wichtig, mögliche Einflussgrößen auf die Kriegsberichterstattung und ihren Umfang im Zeitverlauf zu bestimmen. In Abb. 1 sind die wichtigsten in der Literatur genannten Einflussgrößen entlang eines gedachten Nachrichtenflusses angeordnet. Sie werden im Folgenden näher erläutert. 


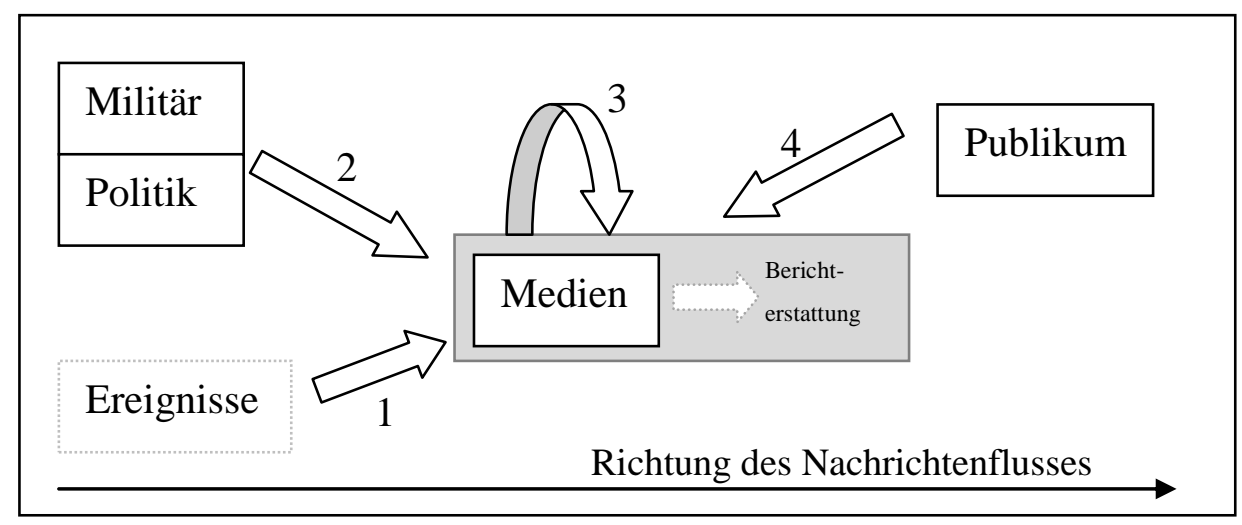

Eigene Darstellung, Adaption nach Löffelholz (2002, S. 36).

(1) Kriege selbst und auch Teil-Ereignisse wie Bombenangriffe werden zu medial berichteten Ereignissen. Eine besondere Ereignisform stellen dabei Schlüsselereignisse dar. Laut Kepplinger (2001, S. 123) zeichnen sie sich durch eine besondere mediale Beachtung aus. Auch in Studien zu Kriegen werden Berichterstattungsspitzen auf bestimmte Ereignisse zurückgeführt wie bspw. bei Krüger (2003, S. 398-400) für den Irak-Krieg 2003. Den Nachrichtenwert von Kriegsereignissen bestimmen dabei vor allem Nachrichtenfaktoren wie Neuigkeit, Betroffenheit, Nähe, Außergewöhnlichkeit, Überraschung, Interesse, Negativität, Personalisierung, Dauer, Eindeutigkeit, Emotionalität sowie Beobachtbarkeit (vgl. Staiger 2004, S. 163-164). Auch konkurrierende externe Ereignisse können die Berichterstattung über einen Krieg beeinflussen. So führt Hils (2007, S. 268-269) den vorübergehenden Einbruch der Berichterstattung amerikanischer Nachrichtenkanäle über den Kosovokrieg im April 1999 auf den Amoklauf in einer USamerikanischen High School in Littleton zurück.

(2) Obwohl sich die Beziehung zwischen Politik/Militär und Medien in westlichen Gesellschaften grundsätzlich als nicht-deterministisch beschreiben lässt (vgl. Löffelholz 2004, S. 20), vollzieht sich Kriegsberichterstattung dennoch in einem „komplizierten Beziehungsquadrat der Krisenkommunikation“"(Löffelholz 2002, S. 36), in dem sich die Medien neben den Bedürfnissen des Publikums auch den Einflüssen von Politik und 
Militär ${ }^{2}$ ausgesetzt sehen. Den Einfluss politischer Eliten auf die Medien formuliert auch Bennetts (1990) Indexing-These, die gerade in Bezug auf die Kriegsberichterstattung wiederholt Unterstützung erfuhr (vgl. z. B. Bennett et al. 2007; Robinson et al. 2009). Und auch der Rally-Effekt, welcher den Popularitätsanstieg von Regierungen im Kriegsfall beschreibt (vgl. Bytzek 2005, S. 369) kann nicht nur aus einer Medienwirkungsperspektive heraus betrachtet werden. Es wurde vielmehr konstatiert, dass Medien vor allem zu Beginn von Kriegen zu einer ähnlichen Kriegsbewertung kommen wie Politiker (vgl. Aday 2010, S. 441) und dass Regierungen im Gegensatz zu ihren Kritikern über ein gewisses Informationsmonopol verfügen, welches sich in der medialen Berichterstattung widerspiegelt (vgl. Brody 1991, S. 62).

Eine Stufe weiter gehen Versuche, in Kriegen die Berichterstattung von Journalisten auf direktem Weg zu beeinflussen. Das so genannte ,Pool-System“ im Irakkrieg von 1991 oder ,eingebettete‘ Journalisten im Irakkrieg 2003 (vgl. Esser et al. 2005, S. 315), aber auch das ,Information Warfare‘ des US-Verteidigungsministeriums (vgl. Dominikowski 2004, S. 76-77) seien beispielhaft für solche Praktiken genannt.

(3) Medien beobachten sich gegenseitig und beeinflussen sich untereinander, da sie in einer publizistischen und ökonomischen Konkurrenz zueinander stehen (vgl. Vliegenthart und Walgrave 2008). Auf die Thematisierung militärischer Ereignisse können sie nicht verzichten, denn diese ist dem wirtschaftlichen Erfolg zuträglich (vgl. Büttner und Kladzinski 2004, S. 223). Andererseits eignet sich „blutige Gewalt nicht als Werbeumfeld“ (Heinrich 2008, S. 202), und so könnte sich mit dem Druck der Werbewirtschaft der Rückgang der Berichterstattung über Kriege bei privaten TV-Sendern erklären lassen, wie er von Krüger (2003, S. 398-400) beobachtet wurde. Auch der Trend zur Selbstbeobachtung und Selbstthematisierung ist gerade in Bezug auf die Kriegsbericht-

\footnotetext{
${ }^{2}$ Die Akteure Militär und Politik sind in Abb. 1 zusammengefasst, da besonders im Kriegsfall von einer engen Ankopplung des Militärs an die Politik ausgegangen wird.
} 
erstattung besonders stark angesehen (vgl. Staiger 2004; Krüger 2003; Esser et al. 2005).

(4) Einflüsse auf die Berichterstattung durch das Publikum sind auf verschiedenen Ebenen denkbar. In Form einer spontanen, unsystematischen Rückkopplung treten Leserbriefe an Journalisten auf, die dann auch teilweise zu Berichterstattung werden, sofern sie bspw. in einer Zeitung abgedruckt werden (vgl. Mast 2008, S. 62). Aufgrund professioneller Publikumsforschung sind ebenso Einflüsse auf die Berichterstattung zu erwarten. In einigen Studien zeigte sich, dass das Publikumsinteresse in Kriegen steigt (vgl. z. B. Imhof 1997, S. 232-236; Grimm 1997, S. 212-218) und die Medien mit zusätzlicher Information darauf reagieren (vgl. Zelizer 1992, S. 68-69). Andererseits konnte mit längerer Dauer der Berichterstattung über einen Krieg das Phänomen der „fatigue“, also die Müdigkeit und das Desinteresse des Publikums, festgestellt werden (vgl. z. B. Ruigrok et al. 2005, S. 175), was zu einem Rückgang der Berichterstattung führen kann.

Die hier vorgestellten Einflussfaktoren zeigen, dass Kriegsberichterstattung ein komplexer Untersuchungsgegenstand ist, der sich nur durch das Zusammenspiel vieler unterschiedlicher Faktoren erklären lässt. Manche der Einflussfaktoren hängen direkt mit dem Faktor Zeit zusammen - wie bspw. das abebbende Zuschauerinteresse im Zeitverlauf oder die Nachrichtenfaktoren Aktualität bzw. Neuigkeit. Doch auch für die übrigen Einflussfaktoren ist zu vermuten, dass die Zeitdimension insofern eine Rolle spielt, als dass ihr Einfluss in unterschiedlichen Phasen der Berichterstattung unterschiedlich stark ist. Überträgt man z. B. Erkenntnisse zum Zusammenspiel von Schlüsselereignissen und Intermedia Agenda-Setting (vgl. Kepplinger und Habermaier 1995; Vliegenthart und Walgrave, 2008; Wien und Elmelund-Præstekær 2009) auf die Kriegsberichterstattung, so liegt nahe, dass sich Medien in ihrer Berichterstattung vor allem bei Ausbruch eines 
Krieges aneinander orientieren, also in ihren Entscheidungen, ob und wieviel sie über einen Krieg berichten.

\section{Dynamische Betrachtung von Berichterstattung}

Die Dynamik medialer Berichterstattung wird greifbar, wenn sie über einen gewissen Zeitraum beobachtet wird und ihre Entwicklung und Veränderung im Zeitverlauf im Fokus der Analyse steht (vgl. Kolb 2005, S. 43). Im Folgenden wird zunächst dargestellt, welche Ansätze zur dynamischen Betrachtung der Kriegsberichterstattung bereits bestehen. Diese werden dann um die kommunikationswissenschaftliche Perspektive der Themenzyklusmodelle ergänzt.

\subsection{Studien zur Phaseneinteilung in der Kriegsberichterstattung}

In einigen Studien zur Kriegsberichterstattung kommt es in der Analyse - wenngleich nicht aus einer zentralen Perspektive heraus - auch zur Beachtung quantitativer Berichterstattungsverläufe (vgl. z. B. Hils 2007, S. 269; Bytzek 2007, S. 107-108; Krüger 2003, S. 398-400). Die genannten Studien lassen gewisse Ähnlichkeiten in den Mustern des Berichterstattungsumfangs über verschiedene Medien hinweg erkennen: So ist die Berichterstattung zu Beginn von Kriegen am stärksten ausgeprägt und geht dann meist kontinuierlich zurück, unterbrochen von Berichterstattungsspitzen zu besonderen Ereignissen. Diese Erkenntnisse sind jedoch wenig systematisiert oder theoretisch untermauert in Bezug auf die beobachteten Berichterstattungsverläufe, da diese auch nicht im Fokus der Analysen standen. Nur wenige Studien verfolgen gezielt die Kriegsberichterstattung in einer Längsschnittperspektive und liefern Anhaltspunkte für eine Phaseneinteilung (vgl. z. B. Shaw und Martin 1993, Fröhlich et al. 2007). Ebenso werden zumeist nur Aussagen über kurze Untersuchungszeiträume gemacht, die sich auf den Beginn eines Krieges sowie die darauf folgenden Wochen konzentrieren. 
In dieser Hinsicht etwas umfassender sind die Beschreibungen von Savarese (1993) und Löffelholz (2003), die sich noch dazu in ihrer zeitlichen Perspektive gut ergänzen. Während Savarases (1993) Analyse sich auf den Berichterstattungsumfang vor einem Krieg beschränkt, macht Löffelholz (2003) Vorschläge zur Einteilung und Benennung von Berichterstattungsphasen nach Kriegsausbruch. Beide Ansätze werden im Folgenden kurz beschrieben.

Savarese (1993, S. 56-58) stellt für die Vorberichterstattung in zehn europäischen Tageszeitungen zum Golf-Krieg 1991 empirisch die Phasen ,surprise', ,interest ${ }^{6}$, ,routine“, waiting' und schließlich ,flood' fest. In der ersten Phase (,surprise'), als Kuwait durch den Irak angegriffen wurde, sowie in der letzten Phase (,flood`) mit Beginn der USgeführten Kriegsoperationen ist die Berichterstattung am umfangreichsten. Dazwischen liegt eine Periode mit geringerem Berichterstattungsumfang, weshalb sich die Vorkriegsberichterstattung durch einen u-förmigen Verlauf auszeichnet.

Bei Löffelholz (2003, S. 11) beginnt die Beschreibung von Phasen der Kriegsberichterstattung mit dem Kriegsausbruch. Für Kriege, die als ,journalistisch bedeutsam“ erachtet werden, wird ein Verlaufsmuster angenommen, dessen Phasen mit den Begriffen ,Monopolisierung', ,Dominierung', ,Normalisierung' sowie ,Marginalisierung' bezeichnet werden. Während der Monopolisierung und Dominierung beherrscht das Topereignis Krieg die Medien, in den folgenden Phasen werden auch andere Themen wieder prominenter berichtet. Für verschiedene Medien kann dabei ein unterschiedlicher Verlauf festgestellt werden, und der Rückgang der Berichterstattung kann durch besonders herausragende Ereignisse unterbrochen werden (vgl. Löffelholz 2007, 2004, 2003). Sowohl Savarese (1993, S. 71) als auch Löffelholz (2008, S. 237-238) vermuten, dass die Verläufe der Kriegsberichterstattung vor allem ereignisgetrieben sind. Dies begründen sie u. a. mit den Erwartungen des Publikums, das im Zeitverlauf das Interesse ver- 
liert und so stets hohe Anforderungen an die Nachrichtenselektion und die Visualisierbarkeit stellt. Aus diesem Grund schafften es lange andauernde Kriege nur noch in Form kleinerer Artikel auf hintere Seiten der Tageszeitung (vgl. Löffelholz 2007, S. 237). Auch Fröhlich et al. (2007) beobachten in ihrer Längsschnittanalyse, dass die Berichterstattung über Kriege besonders an konkrete Kriegsereignisse gekoppelt ist, und vor allem bei Ausbruch eines Krieges deutlich steigt.

Diese Beobachtungen aus Studien zur Kriegsberichterstattung machen deutlich, dass die Übertragung des Themenzyklusmodells auf die Berichterstattung über Kriege naheliegt, und zumindest Savarese und Löffelholz tragen mit ihren ersten Systematisierungsvorschlägen dazu bei. Diese Vorschläge zur Phaseneinteilung der beiden Autoren unterscheiden sich allerdings voneinander, indem sie sich auf jeweils unterschiedliche Zeitabschnitte in der Berichterstattung konzentrieren. Beide Betrachtungsweisen sind für ein Themenzyklusmodell der Kriegsberichterstattung jedoch von Interesse, und so sollte dieses die Ansätze beider Autoren und somit die Berichterstattung vor und nach Kriegsausbrüchen integrieren.

\subsection{Themenzyklusstudien}

In der Kommunikationswissenschaft haben Phasenmodelle, welche die Karriere eines Themas über einen gewissen Zeitraum modellieren und diese in aufeinander folgende Phasen einteilen, bereits eine lange Tradition. Grundlegend für diese Forschungsrichtung sind die viel zitierten theoretischen Arbeiten von Luhmann (1979) und Downs (1972), die den Begriff des Themen- bzw. Aufmerksamkeitszyklus prägten. Themenzyklen können dabei als Berichterstattungsmuster im Zeitverlauf betrachtet werden, die sich in ähnlicher Form wiederholen (vgl. Kolb 2005, S. 47-50). Andere Autoren bezeichnen Thematisierungsverläufe auch als, Themenkarriere' (vgl. Mathes und 
Pfetsch 1991), oder ,Medien-Hype' (vgl. z. B. Vasterman 2005; Wien und ElmelundPræstekær 2009).

Die meisten Studien, welche Thematisierungsverläufe behandeln, teilen den Verlaufszyklus in vier oder fünf Phasen ein (vgl. z. B. Berens 2001; Kolb 2005; Nisbet und Huge 2006; Mathes und Pfetsch 1991). Die empirischen Analysen beschäftigen sich teils mit langfristigen Untersuchungsgegenständen wie der BiotechnologieBerichterstattung (vgl. Nisbet und Huge 2006) oder dem Klimawandeldiskurs (vgl. McComas und Shanahan 1999), teils aber auch mit eng abgegrenzten Gegenständen wie der Debatte um die Volkszählung (vgl. Mathes und Pfetsch 1991), bisher jedoch noch nicht dezidiert mit Kriegen. Unabhängig von der thematischen Ausrichtung lässt sich in den meisten dieser Studien ein typischer Verlauf der Berichterstattung beobachten, der durch die Thematisierungskurve in Abb. 2 schematisch dargestellt wird: Die Berichterstattung zu einem Thema schwankt auf niedrigem Niveau und erste lokale Berichterstattungsmaxima sind möglich. Nach einem Durchbruch des Themas ist ein steiler Anstieg der Berichterstattung zu beobachten. Nach einer meist schmalen Spitze fällt das Niveau der Berichterstattung im weiteren Verlauf - teils stufenweise - wieder deutlich ab.

\section{Abb. 2: Idealtypischer Verlauf von Themenzyklen}

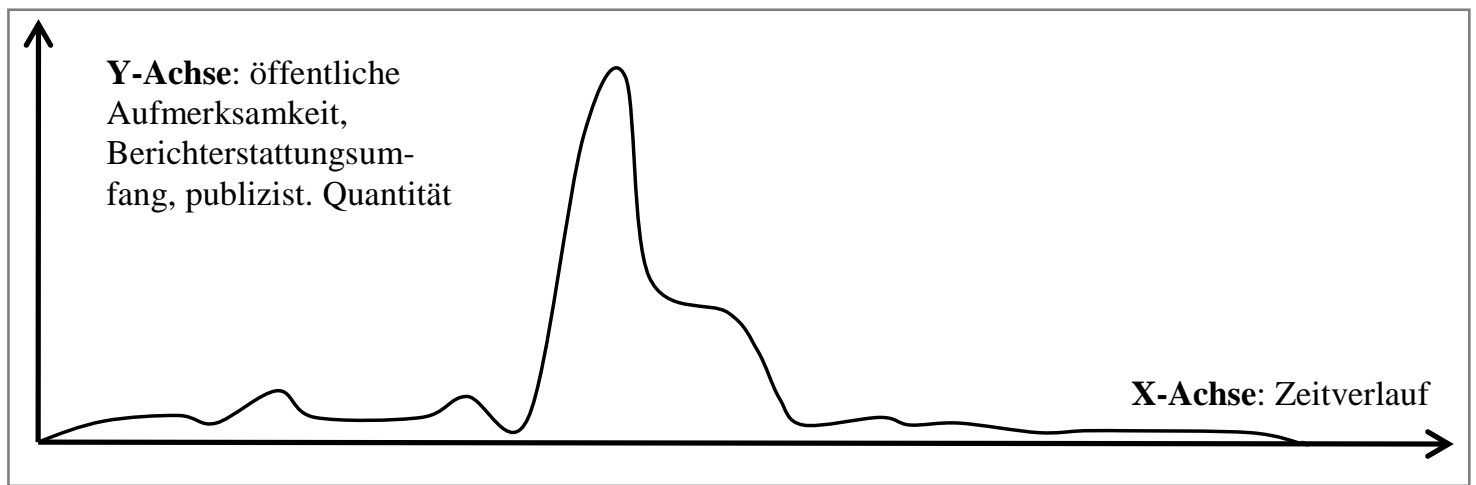

Quelle: Eigene Darstellung in Anlehnung an Kolb 2006, S. 183; Nisbet und Huge 2006, S. 22; Berens 2001, S. 30, 35; Mathes und Pfetsch 1991, S. 58; McComas und Shanahan 1999, S. 43.

Dieses grundlegende Muster findet sich je nach empirischem Untersuchungsgegenstand in unterschiedlichen Ausprägungen. Zum Teil dauert die Aufschwung- (vgl. z. B. Be- 
rens, 2001; Mathes und Pfetsch 1991), zum Teil die Abschwungphase länger (vgl. z. B. Wien und Elmelund-Præstekær 2009). Ebenso werden Themenkarrieren mit mehreren, stark ausgeprägten Berichterstattungsspitzen beobachtet, zwischen denen ruhigere Phasen mit geringer Thematisierung liegen (vgl. Weßler 1999; Franz 2000; Shih et al. 2008). Auch die Länge der betrachteten Zyklen sowie die Länge der Phasen unterscheiden sich je nach Problem und Untersuchungsanlage.

Grundsätzlich deckt sich der in Abb. 2 dargestellte typische Themenzyklus mit den Beschreibungen über den Verlauf der Kriegsberichterstattung (vgl. Abschnitt 2.1). Es macht daher grundlegend Sinn, das klassische Themenzyklusmodell heranzuziehen, um auch für die Kriegsberichterstattung zu modellieren, wie diese sich im Zeitverlauf entwickelt. Allerdings ist auch hier davon auszugehen, dass sich die individuellen Verläufe je nach Krieg deutlich voneinander unterscheiden können. Der Krieg, den die libanesische Armee gegen die islamistische Gruppierung Fatah al-Islam im Sommer 2007 für etwa drei Monate führte, unterscheidet sich in seiner Dauer fundamental von dem Krieg, der mittlerweile seit über 20 Jahren - ohne absehbares Ende - in Somalia geführt wird (vgl. Schreiber 2008, S. 2-5). Will man die Erkenntnisse anderer Studien auf den Bereich der Kriegsberichterstattung übertragen, sind daher weder Modelle, die sich auf kurze und gut abgrenzbare, noch solche, die sich auf längerfristige problembehaftete Themen stützen, generell auszuschließen.

\section{Ein Phasenmodell der Kriegsberichterstattung}

Im Folgenden wird ein Phasenmodell der Kriegsberichterstattung vorgestellt, welches sich zum einen auf das klassische Themenzyklusmodell stützt, zum anderen relevante Unterschiede zwischen Kriegen berücksichtigt und typologisiert. Dabei bezieht es die Charakteristika der Vorhersehbarkeit und der Dauer von Kriegen mit ein. Je nach Kom- 
bination der Dimensionen lassen sich unterschiedliche Berichterstattungsverläufe vermuten (vgl. Tab. 1). In Anlehnung an Löffelholz (2003, S. 11) gilt dabei die Einschränkung, dass es sich um die Verläufe medial beachteter Kriege handeln soll, die also die Schwelle der Veröffentlichung mindestens zu Beginn überschreiten. Aus dieser Annahme lässt sich schließen, dass einem solch medial beachteten Kriegsereignis zumindest anfänglich ein gewisser Nachrichtenwert zugeschrieben wird, was das Vorhandensein bestimmter Nachrichtenfaktoren wie bspw. Neuigkeit, Betroffenheit, Nähe, Überraschung, Eindeutigkeit (vgl. Staiger 2004, S. 163-164) impliziert.

Im Folgenden konzentrieren wir uns auf die Betrachtung der Einflussgrößen Vorhersehbarkeit und Dauer auf die Berichterstattung, die ihrer Terminologie nach der Benennung einzelner in der Literatur beschriebener Nachrichtenfaktoren ähneln oder gar entsprechen. Auch inhaltlich sind diese Einflussgrößen mit Nachrichtenfaktoren in Verbindung zu bringen. Dabei kommt die Vorhersehbarkeit eines Kriegsereignisses dem Nachrichtenfaktor Überraschung am nächsten, wenn es darum geht, ob ein Ereignis erwartbar ist. In Bezug auf die Vorhersehbarkeit unterscheiden Nord und Strömbäck (2003, S. 55) zwischen vorhersehbaren und überraschenden Krisenereignissen. Vorhersehbare Krisen werden medial vorbereitetet und bereits berichtetet (z. B. Kosovokrieg), wohingegen sich die Medien auf überraschende Krisen (z. B. Terroranschläge vom 11. September 2011) nicht vorher einrichten können. Für das Phasenmodell der Kriegsberichterstattung stellt sich die Frage, ob bereits vor einem Kriegsausbruch über einen künftigen Krieg berichtet wird oder nicht; wir nehmen in Rückgriff auf Nord und Strömbäck an, dass vorhergesehene Kriege bereits vor ihrem Ausbruch thematisiert werden, unvorhergesehene dagegen nicht.

Auch die Einflussgröße Dauer lässt sich als Nachrichtenfaktor begreifen, von welcher wir annehmen, dass sie den Verlauf der Berichterstattung nach einem Kriegsausbruch 
maßgeblich beeinflusst, denn mit voranschreitender Zeit ist meist eine gewisse mediale Gewöhnung an ein Ereignis und eine geringer ausgeprägte Berichterstattung zu beobachten. Unsere Annahme stützen wir auch auf die oben erwähnten Studien zu Berichterstattungsverläufen. Hier ließ sich in Studien mit einem kurzfristig abgegrenzten Untersuchungsgegenstand lediglich eine Berichterstattungsspitze beobachten (vgl. z. B. Mathes und Pfetsch 1991). Bei den längerfristigen Untersuchungsgegenständen konnten jedoch mehrere Maxima mit zeitlichen Abständen beobachtet werden (vgl. z. B. Franz 2000; Weßler 1999). Für unser Phasenmodell wollen wir daher zwischen längerfristig, ggf. über Jahre andauernde sowie zwischen kurzen und kurzfristig abgeschlossenen Kriegen unterscheiden; analog erwarten wir für kurz andauernde Kriege lediglich ein lokales Maximum der Berichterstattung, wogegen bei langen, über Jahre oder Jahrzehnte dauernden Kriegen mehrere Berichterstattungsspitzen möglich sind.

Tab. 1: Denkbare Verläufe der Kriegsberichterstattung

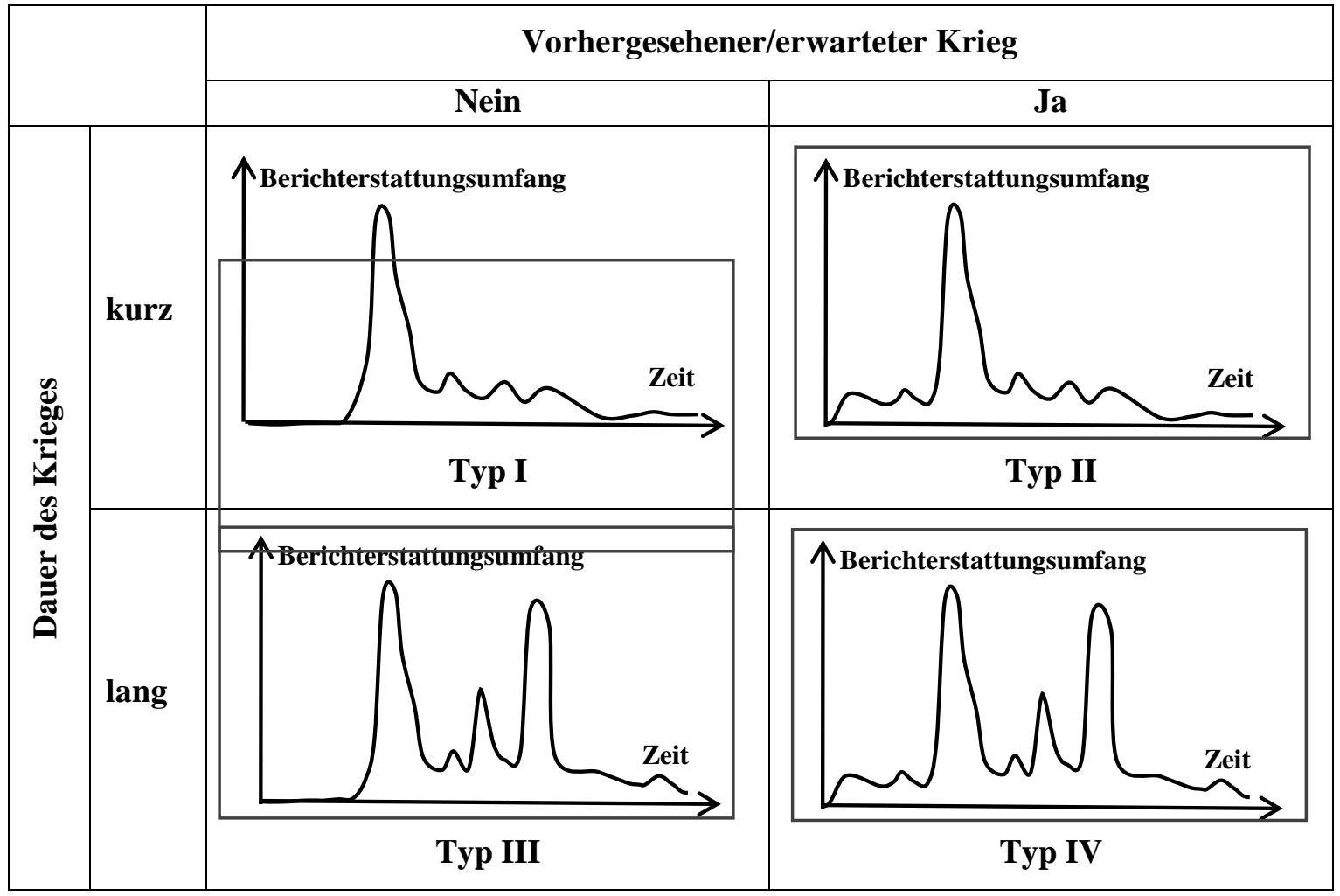


Tab. 1 enthält vier idealtypisch erwartete Verläufe der Kriegsberichterstattung anhand der Dauer und Vorhersehbarkeit eines Krieges. Für einen medial beachteten, kurzen und unerwarteten Krieg (Fall I) ist zu erwarten, dass die Berichterstattung erst mit Kriegsbeginn und Gewalteskalation einsetzt, rasant ansteigt und über einen geringen Zeitraum auf hohem Niveau verharrt, um dann steil abzusinken und nach dem Krieg für eine gewisse Zeit auf höherem Niveau als zuvor zu bleiben. Bei einem vorhersehbaren, länger andauernden Krieg (Fall IV) hingegen dürfte bereits vor Kriegsausbruch vom drohenden Konflikt berichtet werden, und nach einem ersten Berichterstattungsmaximum zu Kriegsbeginn könnte es in der Folge zu weiteren Maxima kommen. Die Berichterstattungskurven der Typen II und III setzen sich entsprechend aus den beschriebenen Elementen der Fälle I und IV zusammen.

In Anlehnung an Kolb (2005, S. 45) lassen sich Berichterstattungsphasen anhand quantitativer Kriterien im Themenzyklus abgrenzen. Abb. 3 enthält die Berichterstattungskurve des Kriegstyp IV mit einem Maximum an sechs Phasen: (1) Durchbruch-, (2) Aufschwung-, (3) Hoch-, (4) Abschwung-, (5) berichtsschwache Zwischen- sowie (6) Marginalisierungsphase. ${ }^{3}$ Nach Phase (5) können erneut Aufschwung-, Hoch- sowie Abschwungphasen (2-4b) auftreten. Für die Berichterstattung zu den anderen Kriegstypen werden jeweils weniger Phasen vermutet.

\footnotetext{
${ }^{3}$ Die Benennung der Phasen erfolgte in Anschluss an die oben benannte Literatur (Phasen 1-4, 6) und nach eigenen Überlegungen (Phase 5).
} 
Abb. 3: Phasenmodell der Kriegsberichterstattung und Phaseneinteilung

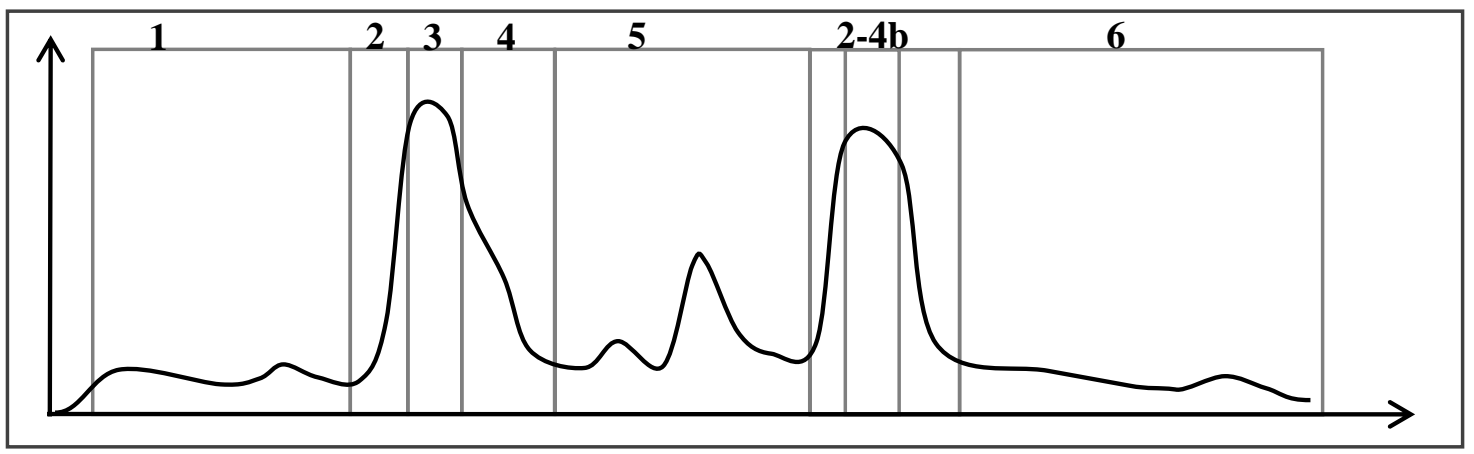

\begin{tabular}{|c|c|c|c|c|c|}
\hline $\begin{array}{l}\text { (1) Durch- } \\
\text { bruch }\end{array}$ & $\begin{array}{l}\text { (2) Auf- } \\
\text { schwung }\end{array}$ & (3) Hoch & $\begin{array}{l}\text { (4) Ab- } \\
\text { schwung }\end{array}$ & $\begin{array}{l}\text { (5) berichts- } \\
\text { schwache Zwi- } \\
\text { schenphase }\end{array}$ & $\begin{array}{l}\text { (6) Marginalisie- } \\
\text { rung }\end{array}$ \\
\hline $\begin{array}{l}\text { Berichter- } \\
\text { stattungsum- } \\
\text { fang } \\
\text { schwankt } \\
\text { kontinuier- } \\
\text { lich auf ge- } \\
\text { ringem Ni- } \\
\text { veau, ohne } \\
\text { starken An- } \\
\text { stieg. }\end{array}$ & $\begin{array}{l}\text { Starker } \\
\text { Anstieg } \\
\text { einige } \\
\text { Zeitab- } \\
\text { schnitte in } \\
\text { Folge, } \\
\text { deutlich } \\
\text { höheres } \\
\text { Niveau } \\
\text { wird er- } \\
\text { reicht. }\end{array}$ & $\begin{array}{l}\text { Etwa } \\
\text { gleichblei- } \\
\text { bend hohes } \\
\text { Niveau der } \\
\text { Berichter- } \\
\text { stattung: } \\
\text { leichte } \\
\text { Schwan- } \\
\text { kungen } \\
\text { möglich. }\end{array}$ & $\begin{array}{l}\text { Starkes Ab- } \\
\text { sinken der } \\
\text { Berichter- } \\
\text { stattung über } \\
\text { mehrere } \\
\text { Zeiteinheiten } \\
\text { hinweg, auf } \\
\text { deutlich } \\
\text { geringeres } \\
\text { Niveau. }\end{array}$ & $\begin{array}{l}\text { Abstieg gebremst, } \\
\text { Niveau der Be- } \\
\text { richterstattung } \\
\text { pendelt sich auf } \\
\text { einem Niveau ein, } \\
\text { das im Vergleich } \\
\text { zum Hoch deut- } \\
\text { lich geringer und } \\
\text { zu Phasen (2) und } \\
\text { (6) erhöht ist. }\end{array}$ & $\begin{array}{l}\text { Niveau der Be- } \\
\text { richterstattung } \\
\text { pendelt sich auf } \\
\text { geringem Ni- } \\
\text { veau ohne große } \\
\text { Schwankungen } \\
\text { ein. }\end{array}$ \\
\hline
\end{tabular}

Zur genauen Bestimmung von Anfang und Ende des Berichterstattungszyklus zu einem

Krieg sollte im Idealfall die ersten bzw. letzten Berichte mit Kriegsbezug herangezogen

werden. Die Bestimmung dieser Punkte dürfte sich jedoch praktisch als schwierig gestalten, wenn anfangs zwar über Ereignisse im Zusammenhang mit einem späteren Krieg berichtet wird, dies jedoch noch keinen expliziten Bezug zum späteren Krieg hat. Auch ein Ende der Berichterstattung kann sich im Extremfall (wie z. B. im Fall des Zweiten Weltkrieges) über Jahrzehnte hinziehen. Eine praktikable Lösung, die hier vorgeschlagen werden soll, ist, sich auf einen „Kernzyklus“ der Berichterstattung zu begrenzen, der sich durch eine gewisse quantitative Intensität sowie Kontinuität auszeichnet. 


\section{Empirische Exploration}

Anhand eines Fallbeispiels wird die entwickelte Systematik nun exemplarisch erprobt. Hierzu wurde mit dem Krieg zwischen Israel und der Hisbollah im Libanon 2006 ein medial stark beachtetes (vgl. Krüger 2007, S. 75-76), kurzes und unvorhersehbares (vgl. Albrecht 2008, S. 161) Kriegsereignis ausgewählt, für das ein Berichterstattungsverlauf des Typ I erwartet wird. Zu diesem Kriegsereignis gibt es zwar erste kommunikationswissenschaftliche Veröffentlichungen (vgl. z. B. Bahador 2008; Klaiber 2012; Konieczny und Verbali 2008; Oehmer 2010), diese analysieren jedoch nicht eingehend den Berichterstattungsverlauf und konzentrieren sich durchgehend lediglich auf die Berichterstattung während des Krieges.

Für die Untersuchung wurden die Qualitätszeitungen Frankfurter Allgemeine Zeitung (FAZ) und Süddeutsche Zeitung (SZ) ausgewählt, da laut Scherer et al. (2005, S. 283) durch die überregionale Ausrichtung der Blätter eine umfangreiche Berichterstattung über internationale Themen wie Krieg erwartet werden kann. Um im Gegensatz zu den eben genannten Studien auch Vor- und Nachkriegsberichterstattung feststellen zu können, wurde der Untersuchungszeitraum großzügig vom 01.01.2006 - 28.02.2007 um den vom 12.07. - 14.08. andauernden Krieg gewählt. Durch Archivsuche gingen für die $F A Z$ 688, für die SZ 685 Artikel in die Analyse ein. ${ }^{4}$

Die Untersuchung fokussiert sich zunächst auf quantitative Aspekte des Berichterstattungsverlaufs und dessen Einteilung in Phasen. In begrenztem Umfang werden jedoch zudem formale (z. B. Genre) und inhaltliche Merkmale (z. B. zu Wort kommende Sprecher) der Berichterstattung analysiert, um mögliche Hinweise auf die Einflussfaktoren des Verlaufs (vgl. Abschnitt 1), auch während einzelner Phasen, identifizieren zu kön-

\footnotetext{
${ }^{4}$ Suchstrategie: F.A.Z.-BiblioNet / http://fazarchiv.faz.net/intranet/biblionet: („Hizbullah“ OR “Libanon“) AND „Israel“; SZ Archiv / http://librarynet.szarchiv.de: („Hisbollah“ OR „Libanon“) AND „Israel“.
} 
nen. Hierzu wurde eine Artikel-Stichprobe mit dem Auswertungsprogramm MAXQDA einer inhaltlichen Analyse unterzogen. Bei einigen Analyse-Aspekten wurde mit zuvor grob festgelegten Kategorien gearbeitet, wiederum andere Kategorien ergaben sich in detaillierterer Form erst im Verlauf der Analyse, wobei sich das Vorgehen an der zusammenfassenden qualitativen Inhaltsanalyse nach Mayring (2003; beschrieben bei Kuckartz 2007, S. 91-96) orientierte. So wurden bspw. die Sprecher zunächst im Text markiert und als Einzelcodes gesammelt. Danach wurden die Codes benannt, und Codes mit der gleichen Benennung zu einer Sprecherkategorie zusammengefasst.

\subsection{Einteilung der Berichterstattung in Phasen}

Für den ausgewählten Krieg nach Typ I (kurz und unvorhersehbar) wird ein Ablauf der Berichterstattung in den vier Phasen Aufschwung, Hoch, Abschwung sowie Marginalisierung angenommen. Abb. 4 zeigt die Anzahl der Artikel mit den Suchbegriffen in beiden Zeitungen auf Wochenniveau aggregiert. Die Berichterstattungsverläufe der beiden Zeitungen ähneln sich sehr stark und gleichen dem unterstellten Ablaufmuster für Kriegstyp I aus Tab. $1 .^{5}$

\footnotetext{
${ }^{5}$ Die Analyse aller 80 vor Kriegsausbruch erschienenen Artikel spricht gegen das Vorhandensein einer Durchbruchphase, da nur in fünf Artikeln ein Bezug zu militärischen Auseinandersetzungen zwischen den späteren Kriegsparteien und somit ein möglicher, konkreter Bezug zum Krieg im Sommer festzustellen ist.
} 
Abb. 4: Berichterstattungsumfang - Anzahl Artikel/Woche je Zeitung

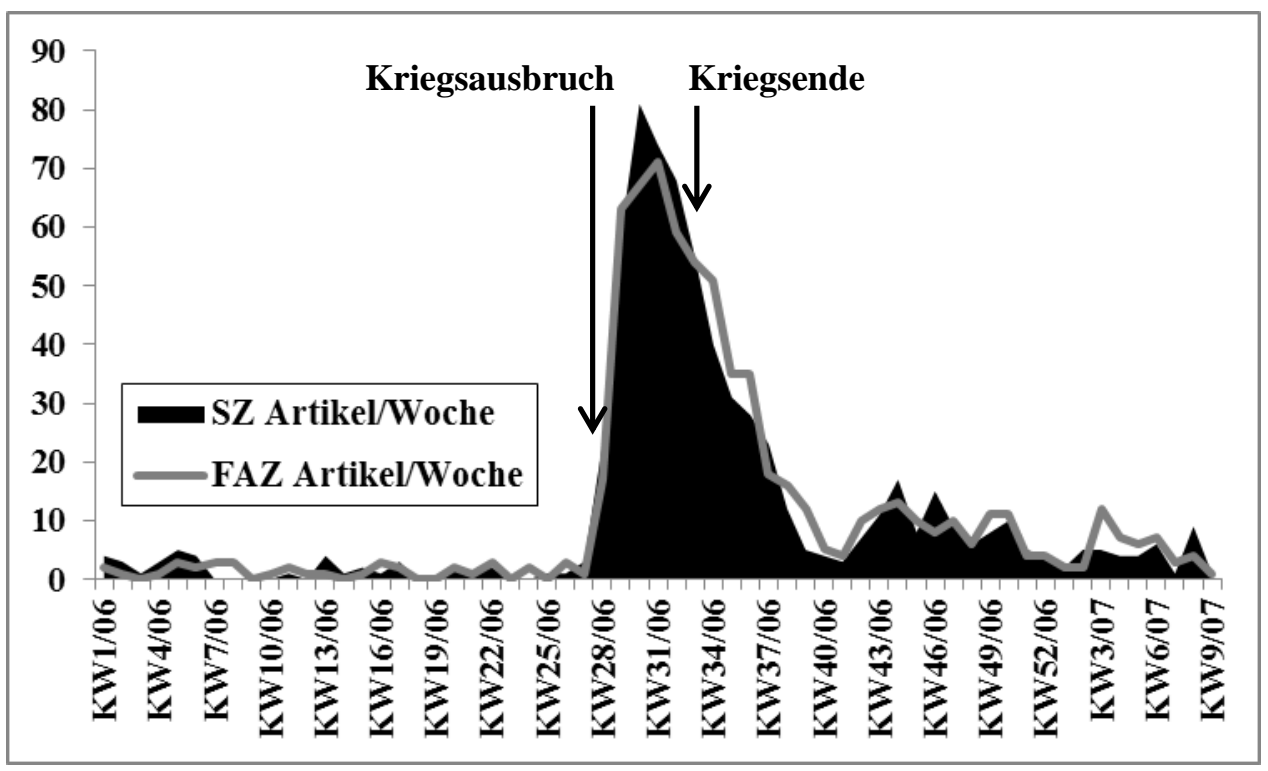

Um die Verläufe in Phasen einzuteilen, wurden hingegen die detaillierteren Umfangsentwicklungen der Artikel von Tag zu Tag sowie das jeweilige Niveau der Berichterstattung auf Tagesebene herangezogen.

Tab. 2: Phaseneinteilung und Anzahl der Artikel sowie Stichprobengrößen je Zeitung

\begin{tabular}{|c|c|c|c|c|c|c|c|}
\hline \multirow{2}{*}{ Medium } & & \multicolumn{4}{|c|}{ Phasen } & \multirow{2}{*}{ gesamt } & \multirow{2}{*}{\begin{tabular}{|l|} 
gesamt ohne \\
Irrelevante
\end{tabular}} \\
\hline & & Aufschwung & Hoch & Abschwung & Marginalisierung & & \\
\hline \multirow{4}{*}{ FAZ } & Dauer & 13.07.-17.07. & $18.07 .-21.08$ & 22.08.-20.09. & 21.09.-28.02. & - & - \\
\hline & Artikel ges. & 28 & 315 & 138 & 169 & 650 & - \\
\hline & Artikel/Tag & 7,0 & 10,9 & 5,3 & 1,3 & 3,4 & - \\
\hline & Stichprobe & 6 & 58 & 31 & 38 & $133(50 \%)$ & $122(50 \%)$ \\
\hline \multirow{4}{*}{ SZ } & Dauer & 13.07.-17.07. & $18.07 .-17.08$ & 18.08.-13.09. & 14.09.-28.02. & - & - \\
\hline & Artikel ges. & 37 & 308 & 129 & 169 & 643 & - \\
\hline & Artikel/Tag & 9,3 & 11,8 & 5,6 & 1,2 & 3,4 & - \\
\hline & Stichprobe & 7 & 72 & 24 & 32 & $135(50 \%)$ & $122(50 \%)$ \\
\hline gesamt & Stichprobe & 13 & 130 & 55 & 70 & $268(100 \%)$ & $244(100 \%)$ \\
\hline Irrelevante & $\begin{array}{l}\text { \# / Anteil an } \\
\text { Stichprobe gesamt }\end{array}$ & $1(7,6 \%)$ & $2(1,5 \%)$ & $6(10,9 \%)$ & $15(21,4 \%)$ & $24(9 \%)$ & - \\
\hline
\end{tabular}

Für die $S Z$ sei dies (analog zu Tab. 2) beispielhaft erläutert: Im Verlauf von vier Tagen steigert sich das Artikelniveau kontinuierlich auf ein stark erhöhtes Niveau. Ein erster Artikelrückgang von 14 auf 7 Artikel pro Tag leitet die knapp einmonatige Hochphase ab dem 18. Juli ein, während der das Artikelniveau um 12 Artikel/Tag schwankt. Dieses Hoch wird von einer Serie von Tagen, an denen die Anzahl der Artikel überwiegend abnimmt und auf ein deutlich niedrigeres Niveau absinkt, beendet. In der nun folgenden 
Abschwungphase liegt das Artikelniveau bei durchschnittlich 5,6 Artikeln/Tag, und der letzte größere Artikelrückgang am 13.09. markiert ihr Ende. Danach folgen nur noch kleinere Schwankungen von Tag zu Tag innerhalb der Marginalisierungsphase (1,2 Artikel/Tag), in der auch immer öfter Tage ganz ohne Artikel, welche die Suchwörter enthielten, registriert werden. Auch bei der $F A Z$ wurden nach analogem Vorgehen ähnliche Anfangs- und Endpunkte der Phasen, sowie (durchschnittliche) Artikelzahlen in den Phasen festgestellt (vgl. Tab. 2).

Wichtig zu erwähnen für die Phaseneinteilung und die nun folgende Ergebnisanalyse ist, dass sich die Berichterstattung in den ersten beiden Phasen überwiegend als direkte Kriegsberichterstattung parallel zu den Kriegsereignissen bezeichnen lässt, während die Abschwungphase bereits ausschließlich Nachkriegsberichterstattung beinhaltet.

\subsection{Analyse der Berichterstattung in den Phasen}

Aus den Artikeln, welche die Suchwörter enthielten, wurde jeder fünfte in die Untersuchungsstichprobe gezogen. Die Artikelstichproben pro Phase und Medium, sowie deren Anteile an der Gesamtstichprobe sind in Tab. 2 enthalten. Aus ihr geht hervor, dass die insgesamt 268 analysierten Artikel sehr gleichmäßig auf $S Z$ (135) und FAZ (133) aufgeteilt sind; auch nach Abzug irrelevanter Artikel bleibt dieses Verhältnis bestehen. Der Anteil irrelevanter Artikel, welche also trotz der enthaltenen Suchwörter keinen Kriegsbezug aufweisen, nimmt besonders in der Marginalisierungsphase mit zunehmendem Abstand zum Kriegsende zu, so dass die in Abb. 4 dargestellten Kurven besonders am Schluss das Berichterstattungsniveau zum Krieg als zu hoch anzeigen dürften. Vor allem für die Aufschwungphase ist außerdem die geringe Fallzahl zu beachten, wenn es um die Aussagekraft der Prozentzahlen in den nun folgenden Tabellen geht. Bezugnehmend zu Abb. 1 werden im Folgenden die Ergebnisse vorgestellt, in denen sich Hinwei- 
se auf die Einflüsse der Faktoren Ereignis, Medien, Publikum sowie Kriegsakteure abzeichnen.

Tab. 3: Ereignisbezug und Artikelanlass der Artikel in den Phasen (Anzahl und prozentual)

\begin{tabular}{|l|c|c|c|c||c|}
\hline \multirow{2}{*}{ Ereignisbezug/Artikelanlass } & \multicolumn{3}{c||}{ Phasen } & \multirow{2}{*}{ gesamt } \\
\cline { 2 - 5 } & Aufschwung & Hoch & Abschwung & Marginalisierung & \\
\hline Ereignisbezug ganzer Artikel/dominant & $7(58,3 \%)$ & $105(82,0 \%)$ & $28(57,1 \%)$ & $13(23,6 \%)$ & $153(62,7 \%)$ \\
\hline Ereignisbezug nicht dominant & $5(41,7 \%)$ & $19(14,8 \%)$ & $17(34,7 \%)$ & $29(52,7 \%)$ & $70(28,7 \%)$ \\
\hline Ereignisbezug am Rande & $0(0 \%)$ & $4(3,1 \%)$ & $4(8,2 \%)$ & $13(23,6 \%)$ & $21(8,6 \%)$ \\
\hline \hline Kriegsbezogener Anlass & $12(80 \%)$ & $119(86,9 \%)$ & $33(71,7 \%)$ & $15(35,7 \%)$ & $179(74,6 \%)$ \\
\hline Anlass außerhalb des Kriegsgeschehens & $1(6,7 \%)$ & $6(4,4 \%)$ & $10(21,7 \%)$ & $21(50 \%)$ & $38(15,8 \%)$ \\
\hline Kein/unklarer Anlass & $2(13,3 \%)$ & $12(8,8 \%)$ & $3(6,5 \%)$ & $6(14,3 \%)$ & $23(9,6 \%)$ \\
\hline
\end{tabular}

Knapp 63 Prozent aller Artikel stellen den Bezug zum Krieg im ganzen Text oder einem Großteil davon her. Im Rest der Artikel werden auch Bezüge zu anderen Ereignissen oder Themen hergestellt und der Krieg ist nur eines von mehreren berichteten Ereignissen (29\%), oder der Bezug zum Krieg ist gar nur am Rande erwähnt (knapp 9\%; vgl. Tab. 3). Im Zeitverlauf sinkt der Anteil der Artikel, die sich dominant mit dem Krieg befassen, und es steigt der Anteil der Artikel, die den Krieg als eines unter mehreren Themen oder lediglich am Rande abhandeln. Diese Ergebnisse erscheinen plausibel, als mit Ende des Krieges nicht mehr über direkte Kriegshandlungen berichtet werden kann, sich die Berichterstattung aber weiterhin mit dem zuvor stattgefundenen Krieg und seinen Folgeereignissen wie der Stationierung der UNIFIL-Truppe im Libanon ab September oder der Diskussion um den Einsatz der deutschen Marine vor der libanesischen Küste befasst. Analog dazu liegen besonders in den Phasen des Abschwungs und der Marginalisierung die Berichterstattungsanlässe immer häufiger außerhalb des direkten Kriegsgeschehens, da diese sich meist auf andere in den Artikeln berichtete Themen beziehen (Anlässe waren bspw. die israelische Innenpolitik, die Regierungskrise im Libanon oder internationale Gipfeltreffen). So hat der Anlass, der den Artikel auslöste, in der letzten Phase in über der Hälfte der Artikel nichts mehr mit dem Krieg zu tun (vgl. Tab. 3). 
Die Berichterstattung ist also eng mit dem Kriegsereignis verbunden und von ihm beeinflusst: Mit Beginn des Krieges setzt die Berichterstattung ereignisgetrieben ein, bleibt während des Krieges intensiv und behält auch nach Kriegsende, besonders in der recht lange andauernden Abschwungphase, noch eine gewisse Bedeutung. Die Artikel mit direktem Kriegsbezug und kriegsbezogenem Anlass werden jedoch seltener, und der Krieg nimmt innerhalb der Artikel immer weniger Platz ein.

Der Einfluss des Publikums und anderer Medien auf die Berichterstattung lässt sich zunächst über die Texttypen bzw. das Genre der Artikel bemessen. Knapp 7 Prozent aller Texte stellen sich als Leserbriefe heraus, und fast 11 Prozent aller Texte sind den Rubriken „Stimmen der anderen“ (FAZ) bzw. „Blick in die Presse“ (SZ) zuzuordnen. Somit wird deutlich, dass ein beträchtlicher Anteil von fast einem Fünftel (Leserbriefe und Pressespiegel zusammen 18,1\%) aller analysierten Artikel auf Leser zurückzuführen oder direkt anderen Zeitungen entnommen ist. Auch hier ist der Anteil der Artikel in den ersten beiden Phasen am höchsten und nimmt dann ab. Die Berichterstattung der Zeitungen orientiert sich also in der Hochphase der Berichterstattung verstärkt an anderen Medien, enthält dadurch auch mehr Fremdmeinungen und bildet ein größeres Meinungsspektrum ab.

Der Einfluss einzelner Akteursgruppen auf den Umfang der Berichterstattung lässt sich indirekt auch daran bemessen, ob und wenn ja wie stark sie in der Berichterstattung zu Wort kommen. Auch hier erhalten die Medien relevante Aussagenanteile (insg. 17,1\%, vgl. Tab. 4), wobei ihr Sprechanteil in der Hochphase mit fast $20 \%$ am größten ist. Dies hängt einerseits direkt mit den oben aufgeführten Artikel-Genres zusammen: Besonders die in anderen Medien veröffentlichten Meinungen zum Konflikt werden verstärkt wiedergegeben, die Medien beobachten sich selbst. Andererseits ist der Wert aber auch darauf zurückzuführen, dass in der Hochphase der Konflikt verstärkt selbst durch $F A Z$ 
und $S Z$ kommentiert wurde. Aus diesen Beobachtungen lassen sich Indizien dafür ableiten, dass die Berichterstattung in ihrer Hochphase verstärkt mediengetrieben war. Das Publikum kommt mittels Leserbriefschreiber insgesamt in unter drei Prozent aller Fälle und damit recht selten zu Wort, was darauf zurückzuführen sein mag, dass pro Leserbrief meist nur eine Aussage erfasst wurde.

Aus Tab. 4 geht hervor, dass Akteure aus dem Bereich der Politik während des gesamten Themenzyklus am häufigsten zu Wort kommen (56,2\%). Zusammengenommen mit dem militärischen Bereich erreichen beide Akteursgruppen einen Aussagenanteil von fast zwei Dritteln. Dies unterstützt zunächst einmal die These, dass gerade diese beiden Akteursgruppen einen besonderen Einfluss auf und Zugang zur Kriegsberichterstattung haben, und die Medien ihre Botschaften häufig in der Berichterstattung wiedergeben. Ausgerechnet in der Hochphase ist der Anteil an Politikeraussagen jedoch am geringsten und das Sprecherspektrum - wie oben bereits erwähnt - am vielseitigsten.

Tab. 4: Sprecher pro Phase nach Kategorien (Anzahl und prozentual)

\begin{tabular}{|c|c|c|c|c||c|}
\hline \multirow{2}{*}{ Sprecherkategorie } & \multicolumn{3}{c||}{ Phasen } & \multirow{2}{*}{ gesamt } \\
\cline { 2 - 5 } & Aufschwung & Hoch & Abschwung & Marginalisierung & \\
\hline Politik gesamt & $25(59,5 \%)$ & $216(49,7 \%)$ & $106(72,6 \%)$ & $37(61,7 \%)$ & $384(56,2 \%)$ \\
\hline Politik Kriegsakteure (Israel/Libanon) & $3(7,1 \%)$ & $57(13,1 \%)$ & $22(15,1 \%)$ & $8(13,3 \%)$ & $90(13,2 \%)$ \\
\hline Militär gesamt & $4(9,5 \%)$ & $43(9,9 \%)$ & $8(5,5 \%)$ & $4(6,7 \%)$ & $59(8,6 \%)$ \\
\hline Militär Kriegsakteure (Israel/Libanon) & $4(9,5 \%)$ & $39(9,0 \%)$ & $7(4,8 \%)$ & $4(6,7 \%)$ & $54(7,9 \%)$ \\
\hline Medien gesamt & $6(14,3 \%)$ & $\mathbf{8 3}(19,1 \%)$ & $20(13,7 \%)$ & $\mathbf{8}(13,3 \%)$ & $117(17,1 \%)$ \\
\hline Bürger/Zivilgesellschaft gesamt & $7(16,7 \%)$ & $\mathbf{8 2}(18,9 \%)$ & $11(7,5 \%)$ & $11(18,3 \%)$ & $111(16,3 \%)$ \\
\hline Leserbriefschreiber & $0(0 \%)$ & $12(2,8 \%)$ & $4(2,7 \%)$ & $1(1,7 \%)$ & $17(2,5 \%)$ \\
\hline Wirtschaft/Sonstige gesamt & $0(0 \%)$ & $11(2,5 \%)$ & $1(0,7 \%)$ & $\mathbf{0 , 0 \%}$ & $12(1,8 \%)$ \\
\hline \hline Gesamt & $42(100 \%)$ & $435(100 \%)$ & $146(100 \%)$ & $60(100 \%)$ & $683(100 \%)$ \\
\hline \hline Politik/Militär der Kriegsakteure & $7(16,7 \%)$ & $96(22,1 \%)$ & $29(19,9 \%)$ & $12(20,0 \%)$ & $144(21,1 \%)$ \\
\hline
\end{tabular}

Gerade im politisch-militärischen Bereich sollte nun zwischen den direkt am Krieg beteiligten Akteuren (sie sind in Tab. 4 separat ausgewiesen) und den restlichen Akteuren aus den betreffenden Sprechergruppen unterschieden werden. Hierbei zeichnet sich folgendes Bild ab: Auf die Politiker und Militärs der direkten Kriegsakteure (israelisches Militär/Regierung, libanesische Regierung/Hisbollah) entfallen mit zusammen etwa 21 Prozent etwas mehr als ein Fünftel aller Aussagen zum Krieg - diese sind für die Presse 
während aller vier Phasen willkommene Informationsquelle mit beträchtlichen Redeanteilen (vgl. Tab. 4, unterste Zeile). Hierbei fällt auf, dass ihr Redeanteil in der Hochphase der Berichterstattung (und damit der Kampfphase des Krieges sowie dem direkten Ereignisbezug), bedingt durch einen größeren Sprechanteil der Militärs, am höchsten ausfällt. Weiterhin ist augenfällig, dass innerhalb der Akteurskategorie Militär fast ausschließlich Sprecher der direkten Konfliktparteien zu Wort kommen, was damit zu erklären ist, dass keine sonstigen militärischen Akteure in die Kampfhandlungen des Konfliktes verwickelt waren. Auf die politischen Sprecher der Kriegsparteien entfallen etwa 13\% aller Aussagen. Besonders in der Abschwung- und Marginalisierungsphase werden die politischen Kriegsakteure relativ gesehen wieder wichtiger, wobei hier vor allem die israelische Regierung auf die meisten Redeanteile kommt.

Innerhalb der übergeordneten Sprecherkategorie ,Politik' entfällt jedoch nur ein knappes Viertel der Aussagen auf die Kriegsakteure. Selbst in der Hochphase des Konfliktes entfallen viele Aussagen auf hochrangige Regierungsvertreter anderer - vor allem westlicher - Staaten, sowie Vertreter internationaler Gremien wie bspw. der UNO oder der Arabischen Liga, die um eine Beendigung des Konfliktes bemüht waren. Diese Beobachtung kann auch in der Abschwung- und Marginalisierungsphase gemacht werden: Hier kommen zunächst verstärkt Vertreter der deutschen Regierung, später dann auch Parteipolitiker (aufgrund der Debatte um die Entsendung deutscher Truppen im Rahmen einer UN-Mission vor die libanesische Küste) sowie UN-Vertreter zu Wort. Die beobachteten Werte sind somit einerseits durch den geänderten Ereignishintergrund, aber auch damit zu erklären, dass die deutschen Medien $S Z$ und $F A Z$ verstärkte Bezüge zur deutschen Innenpolitik herstellten und somit gehäuft deutsche Politiker zu Wort kommen ließen. In diesem Zusammenhang deutet sich dann auch an, dass weitere (Nachrichten-)Faktoren in die Analyse von Berichterstattungsverläufen einbezogen werden 
sollten, denn die erhöhte Berichterstattung zum Krieg über dessen Ende hinaus und die Struktur der in der Berichterstattung zu Wort kommenden Sprecher lassen sich eben dadurch erklären, dass sich für die deutsche Öffentlichkeit durch die Beteiligung an der UN-Mission eine erhöhte Betroffenheit und Relevanz ergab.

Eine weitere Sprechergruppe, die während der gesamten Zeitspanne mit knapp 16\% relevante Aussageanteile auf sich vereinen kann, sind Bürger und Vertreter der Zivilgesellschaft wie bspw. Nichtregierungsorganisationen. Ihr Einfluss kommt zunächst durch den erhöhten Anteil von Gastkommentaren während der ersten beiden und der letzten Phase zu Stande, in denen externen Einzelakteuren die Möglichkeit gegeben wurde, ihre Meinung zum Krieg zu äußern. Dies, und auch die Tatsache, dass während der Hochphase des Krieges verstärkt Betroffene und Opfer des Krieges in Artikeln zu Wort kamen, führt dazu, dass insbesondere hier die Stimmenvielfalt am größten ist. Hier waren die untersuchten Tageszeitungen offenbar darum bemüht, ein möglichst großes Ensemble an Sprechern zu Wort kommen zu lassen.

Insgesamt zeigen die Ergebnisse, dass die Berichterstattung vor allem durch die Ereignisse beeinflusst wird. Dies wirkt sich auch darauf aus, wann welche Sprecher in der Berichterstattung zu Wort kommen. So kamen den Akteursgruppen Medien, Publikum, sowie Politik und Militär der Kriegsparteien, deren Einfluss auf die Berichterstattung hier vor allem interessierte, zusammen genommen beträchtliche Sprechanteile von insgesamt ca. $41 \%$ zu. Vor allem während der kriegerischen Auseinandersetzung und damit der Hochphase der Berichterstattung ist der Anteil der direkt in den Krieg involvierten Akteure im Vergleich zum Durchschnitt über alle Phasen erhöht, was also als Einfluss dieser Akteursgruppen auf den Umfang der Kriegsberichterstattung gedeutet werden kann. Und auch der Sprechanteil der Medien ist in dieser Phase, bedingt durch die erhöhte journalistische Selbstbeobachtung und Kommentierung am höchsten. Von einer 
Dominanz dieser Akteursgruppen kann angesichts eines verbleibenden Sprecheranteils von gut $60 \%$ sowie der größten Sprechervielfalt während der Hochphase jedoch nicht ausgegangen werden.

\section{Schlussfolgerungen und Ausblick}

Wie durch das Phasenmodell der Kriegsberichterstattung unterstellt, zeigte sich für den Krieg im Nahen Osten 2006 ein Ablauf der Berichterstattung in vier Phasen, wie in Verlaufstyp I der Berichterstattung angenommen. Dabei gab es vor dem unvorhergesehenen Kriegsereignis keine Berichterstattung in den beiden untersuchten Zeitungen, nach Beendigung des Krieges jedoch eine noch länger andauernde Berichterstattung mit Kriegsbezug. Die inhaltliche Exploration ergab, dass während des Krieges meist direkte Kriegsereignisse als Artikelanlass dienten, während dies danach nicht mehr so häufig der Fall war. Gleiches gilt für den Bezug der Artikel zum Kriegsereignis - dieser sank nach Kriegsende. Insgesamt kann im vorliegenden Fall von einer sehr ereignisgetriebenen Berichterstattung ausgegangen werden, in deren Umfang sich die Kriegsereignisse direkt widerspiegeln.

Gleichzeitig wird aber auch der Einfluss der Akteursgruppen aus dem Beziehungsgeflecht der Kriegsberichterstattung deutlich (vgl. Abschnitt 1). Auf die direkten Kriegsparteien entfielen knapp ein Fünftel, und ergänzt um das Publikum und die Medien, zusammen ca. $41 \%$ der Sprechakte. Während der Hochphase der Berichterstattung war die Stimmenvielfalt der Akteure insgesamt am größten, zugleich war jedoch auch der Sprechanteil der am Krieg beteiligten Akteure leicht erhöht. Es lässt sich also folgern, dass der beobachtete Themenzyklus nicht rein ereignisinduziert ablief, sondern v.a. in der Hochphase auch akteurs- und mediengetrieben war. Letzteres manifestierte sich vor allem in der verstärkten Wiedergabe alternativer Meinungen (Gastkommentierung) sowie der Reflexion der Inhalte anderer Medien. 
Über den ganzen Themenzyklus hinweg kann nicht von einer Dominanz der kriegsbezogenen Akteure gesprochen werden. Vielmehr zeigt sich, dass andere politische Sprecher (z. B. deutsche Regierungsvertreter und sonstige Politiker sowie Regierungsvertreter von Drittstaaten, UN-Akteure) den Großteil der Aussagen auf sich vereinen. Dass der Einfluss der kriegsbezogenen Akteure im Vergleich zu anderen politischen Akteuren gar nicht so stark ist, lässt sich sicher auch dadurch erklären, dass Deutschland nicht selbst als Kriegsakteur am Libanonkrieg beteiligt war. Es ist zu vermuten, dass die Kriegsakteure in der Berichterstattung eines Landes, das selbst stärker in den Krieg involviert ist, viel größeres Gewicht einnehmen.

Insgesamt hat die vorliegende Studie gezeigt, dass sich auch in der Kriegsberichterstattung klassische Themenzyklen beobachten lassen und es sich folglich lohnt, solche Modelle auf die mediale Beachtung von Kriegen zu übertragen. Dies legen sowohl die Ergebnisse bisheriger Untersuchungen als auch die hier beschriebene explorative Studie nahe. Aufgrund der unterschiedlichen Vorhersehbarkeit sowie der unterschiedlichen Länge von Kriegen sind jedoch auch relevante Abweichungen zum typischen Themenverlauf zu erwarten. So zeigte die Analyse der Berichterstattung zum Libanonkrieg ein Beispiel für einen klassischen eingipfligen Themenzyklus, allerdings ohne latente Phase, da es zu diesem überraschenden Krieg keine Vorberichterstattung gab. Für Kriege von längerer Dauer sind mehrgipflige Themenkarrieren zu vermuten.

Zudem wurde anhand der explorativen Studie demonstriert, dass die zuvor im Modell vorgeschlagene Phaseneinteilung prinzipiell auch für die dynamische Betrachtung von Kriegsberichterstattung Sinn macht. In unterschiedlichen Phasen zeichnet sich ein unterschiedlich großer Einfluss verschiedener Ereignistypen und Akteursgruppen ab. Dies zeigt, dass sich die Berichterstattung dynamisch im Zeitverlauf ändert und daher eine 
Längsschnittuntersuchung von Kriegsberichterstattung im Vergleich zu einer reinen Querschnittperspektive zusätzlichen Erkenntnisgewinn bringt.

Die hier vorgestellte empirische Fallstudie kann jedoch, auch aufgrund des begrenzten Umfangs inhaltlicher Variablen der Berichterstattung, nur erste Anhaltspunkte für die Angemessenheit des vorgeschlagenen Phasenmodells geben. Wir können jedoch durch das Modell generell aufzeigen, welche Perspektiven sich durch eine primär zeitliche Betrachtung für die Erforschung der Kriegsberichterstattung auftun. Eine Betrachtung weiterer Kriege und somit auch der verschiedenen skizzierten Berichterstattungstypen (z. B. Kriege mit Vorberichterstattung oder länger andauernde Kriege mit berichtsschwachen Zwischenphasen) muss in weiteren empirischen Studien erfolgen. Hier sollte dann auch ein verstärkter Fokus auf die Erforschung bestimmter Einflussfaktoren wie Akteure und Ereignisse in den bestimmten Phasen erfolgen. In diesem Zusammenhang stellt sich insbesondere die Frage, inwiefern weitere Nachrichtenfaktoren von Kriegen neben den hier betrachteten Merkmalen Dauer und Vorhersehbarkeit - einen Einfluss auf die Dynamik der Berichterstattung haben. Denn je nach örtlicher Betroffenheit, Kriegsbeteiligung eines Landes und seiner handelnden Akteure dürfte von unterschiedlichen Berichterstattungsverläufen auszugehen sein. Deshalb sollte die Betrachtung von Kriegsberichterstattung auch in ländervergleichender Perspektive erfolgen und sowohl Medien in an einem Krieg beteiligten sowie unbeteiligten Ländern einschließen.

Auch gilt es nach dem Vorbild von Fröhlich et al. (2007) genauer zu untersuchen, wie sich die Kriegsberichterstattung über die Zeit inhaltlich verändert: Wann bekommen z. B. kritische Bewertungen und alternative Deutungsmuster ein stärkeres Gewicht? Ebenso sollte die Untersuchung auf andere Medientypen ausgeweitet werden, um nachzuprüfen, ob auch dort ein ähnlicher Verlauf der Berichterstattung beobachtet werden kann. 
Für den konkreten Aufbau weiterer Analysen wären auch die Untersuchung von Berichterstattung über Konkurrenzereignisse und ihr Einfluss auf die Kriegsberichterstattung interessant. Lohnenswert wären ebenso Publikumsanalysen sowie Analysen zu direkten Medienquellen in Form von Input-Output-Analysen, um genauer bemessen zu können, aus welchen Quellen Journalisten wie wählen, und was letztendlich tatsächlich Eingang in die Berichterstattung findet. 


\section{Literatur}

Aday, S. (2010). Chasing the bad news: An analysis of 2005 Iraq and Afghanistan war coverage on NBC and Fox News Channel. Journal of Communication, 60, 144-164.

Albrecht, M. (2008). Libanon. In W. Schreiber (Hrsg.), Das Kriegsgeschehen 2006. Daten und Tendenzen der Kriege und bewaffneten Konflikte (S. 161-169). Wiesbaden: VS.

Bahador, B. (2008). Framing the 2006 Israel-Hezbollah war? Paper presented at the ISA's 49th Annual Convention, Bridging Multiple Divides, San Francisco, March. http://www.allacademic.com/meta/p251892_index.html. Zugegriffen: 10. Aug. 2009.

Bennett, L. W. (1990). Toward a theory of press-state relations in the United States. Journal of Communication, 40, 103-125.

Bennett, L. W., Lawrence, R.G., \& Livingston, S. (2007). When the press fails: Political power and the news media from Iraq to Katrina. Chicago: University of Chicago Press.

Berens, H. (2001). Prozesse der Thematisierung in publizistischen Konflikten. Ereignismanagement, Medienresonanz und Mobilisierung der Öffentlichkeit am Beispiel von Castor und Brent Spar. Wiesbaden: Westdeutscher.

Brody, R. A. (1991). Assessing the President. The Media, Elite Opinion, and Public Support. Stanford: Stanford University Press.

Brosius, H.-B., \& Eps, P. (1993). Verändern Schlüsselereignisse journalistische Selektionskriterien? Framing am Beispiel der Berichterstattung über Anschläge gegen Ausländer und Asylanten. Rundfunk und Fernsehen, 41, 512-530.

Büttner, C., \& Kladzinski, M. (2004). Krieg und Medien - Zwischen Information, Inszenierung und Zensur. Der Bürger im Staat, 54, 223-228.

Bytzek, E. (2007). Ereignisse und ihre Wirkung auf die Popularität von Regierungen. Von der Schleyer-Entführung zur Elbeflut. Baden-Baden: Nomos.

Bytzek, E. (2005). Kosovokrieg, Kriegsberichterstattung und die Popularität der deutschen Regierungsparteien und -politiker. Medien \& Kommunikationswissenschaft, 53, 369-388. 
Dominikowski, T. (2004). Massenmedien und Massenkrieg. Historische Annäherungen an eine unfriedliche Symbiose. In M. Löffelholz, (Hrsg.), Krieg als Medienereignis II. Krisenkommunikation im 21. Jahrhundert (S. 59-80). Wiesbaden: VS.

Downs, A. (1972). Up and down with ecology - the ,issue-attention cycle”. Public Interest, 28, $38-50$.

Eilders, C., \& Hagen, L. M. (2005). Kriegsberichterstattung als Thema kommunikationswissenschaftlicher Forschung. Ein Überblick zum Forschungsstand und den Beiträgen in diesem Themenheft. Medien \& Kommunikationswissenschaft, 53, 205-221.

Entman, R. M. (2003). Cascading Activation: Contesting the White House's Frame After 9/11. Political Communication, 20, 415-432.

Esser, F., Schwabe, C., \& Wilke, J. (2005). Metaberichterstattung im Krieg. Wie Tageszeitungen die Rolle der Nachrichtenmedien und der Militär-PR in den Irakkonflikten 1991 und 2003 framen. Medien \& Kommunikationswissenschaft, 53 , 314-332.

Franz, B. (2000). Öffentlichkeitsrhetorik. Massenmedialer Diskurs und Bedeutungswandel. Wiesbaden: Deutscher Universitäts-Verlag.

Fröhlich, R. (2010). Research Note: The Coverage of War: Do Women Matter? A Longitudinal Content Analysis of Broadsheets in Germany. European Journal of Communication, 25, $59-68$.

Fröhlich, R., Scherer, H., \& Scheufele, B. (2007). Kriegsberichterstattung in deutschen Qualitätszeitungen. Eine inhaltsanalytische Langzeitstudie zu Framingprozessen. Publizistik, $52,11-32$.

Grimm, J. (1997). Informationsleistungen von Medien in Krisenzeiten. Anomalien des Zuschauerverhaltens während des Golfkriegs. In C. Barth, \& C. Schröter (Hrsg.), Radioperspektiven. Strukturen und Programme (S. 211-229). Baden-Baden: Nomos.

Heinrich, J. (2008). Den Krieg verkaufen. Wirtschaftliche Abhängigkeiten der Berichterstattung. In M. Löffelholz, C. F. Trippe, \& A. C. Hoffmann (Hrsg.), Kriegs- und Krisenberichterstattung. Ein Handbuch (S. 201-202). Konstanz: UVK. 
Hils, J. (2007). Manipuliertes Volk? Mediendemokratie und die militärische Interventionspolitik der USA am Beispiel der Kosovokriege. Baden-Baden: Nomos.

Imhof, C. (1997). Fernseh- und Radionutzung in politischen Krisenzeiten. In C. Barth, \& C. Schröter (Hrsg.), Radioperspektiven. Strukturen und Programme (S. 231-238). BadenBaden: Nomos.

Iyengar, S., \& Simon, A. (1994). News Coverage of the Gulf Crisis and Public Opinion: A Study of Agenda-Setting, Priming, and Framing. In W. L. Bennett, \& D. L. Paletz (Hrsg.), Taken by Storm: The Media, Public Opinion, and U.S. Foreign Policy in the Gulf War (S. 167-185). Chicago: The University of Chicago Press.

Kepplinger, H. M. (2001). Der Ereignisbegriff in der Publizistikwissenschaft. Publizistik, 26, $117-139$.

Kepplinger, H. M., \& Habermeier, J. (1995). The impact of key events on the presentation of reality. European Journal of Communication, 10, 371-390.

Klaiber, S. (2012). Der Libanonkrieg 2006. Eine Inhaltsanalyse der Berichterstattung in The Daily Star Lebanon, The Jerusalem Post und der Süddeutschen Zeitung auf Basis der Framing-Theorie. In K.-D. Altmeppen, \& R. Greck (Hrsg.), Facetten des Journalismus. Theoretische Analysen und empirische Studien (S. 201-219). Wiesbaden: Springer.

Kolb, S. (2005). Mediale Thematisierung in Zyklen. Theoretischer Entwurf und empirische Anwendung. Köln: Herbert von Halem.

Konieczny, E., \& Verbali, M. (2008). Kommunikationsereignisanalyse „Libanonkrieg“. Berichterstattungsdynamik und Darstellung der Konfliktparteien. fög research papers 2008-0001. Zürich: fög - Forschungsbereich Öffentlichkeit und Gesellschaft.

Krüger, U. M. (2007). InfoMonitor 2006: Fernsehnachrichten bei ARD, ZDF, RTL und SAT.1. Strukturen, Themen und Politikerpräsenz. Media Perspektiven (2), 58-82.

Krüger, U. M. (2003). Der Irak-Krieg im deutschen Fernsehen. Analyse der Berichterstattung in ARD/DasErste, ZDF, RTL und SAT.1. Media Perspektiven (9), 398-413.

Kuckartz, U. (2007). Einführung in die computergestützte Analyse qualitativer Daten (2. Aufl.). Wiesbaden: VS. 
Löffelholz, M. (2008). Hintergründe ausgeblendet. Wie Medien über Kriege (nicht) berichten. In M. Löffelholz, C. F. Trippe, \& A. C. Hoffmann (Hrsg.), Kriegs- und Krisenberichterstattung. Ein Handbuch (S. 236-240). Konstanz: UVK.

Löffelholz, M. (2007). Grundlagen einer medienorientierten Krisenkommunikation. Beziehungen zwischen Sicherheitspolitik, Militär und Öffentlichkeit in unterschiedlichen Konfliktphasen. In H.-V. Hoffmann (Hrsg.), Netzwerk Kommunikation in Zeiten der Krise (S. 221-246). Baden-Baden: Nomos.

Löffelholz, M. (2004). Krisen- und Kriegskommunikation als Forschungsfeld. Trends, Themen und Theorien eines hoch relevanten, aber gering systematisierten Teilgebietes der Kommunikationswissenschaft. In M. Löffelholz (Hrsg.), Krieg als Medienereignis II. Krisenkommunikation im 21. Jahrhundert (S. 13-55). Wiesbaden: VS.

Löffelholz, M. (2003). Distanz in Gefahr. journalist,(5), 10-12.

Löffelholz, M. (2002). Krisenkommunikation. Die Forschung boomt. message (1), 36-39.

Luhmann, N. (1979). Öffentliche Meinung. In W. R. Langenbucher (Hrsg.), Politik und Kommunikation. Über die öffentliche Meinungsbildung (S. 29-61). München: Piper.

Mast, C. (2008). ABC des Journalismus. Ein Handbuch (11. Aufl.). Konstanz: UVK.

Mathes, R., \& Pfetsch, B. (1991). The Role of the Alternative Press in the Agenda-Building Process: Spill-over Effects and Media Opinion Leadership. European Journal of Communication, 6, 33-62.

Mayring, P. (2003). Qualitative Inhaltsanalyse. Grundlagen und Techniken (8. Aufl.). Weinheim: Deutscher Studien Verlag.

McComas, K., \& Shanahan, J. (1999). Telling Stories About Global Climate Change: Measuring the Impact of Narratives on Issue Cycles. Communication Research, 26, 30-57.

Nisbet, M. C., \& Huge, M. (2006). Attention Cycles and Frames in the Plant Biotechnology Debate: Managing Power and Participation through the Press / Policy Connection. The Harvard International Journal of Press/Politics, 11(2), 3-40.

Nord, L. W., \& Strömbäck, J. (2003). Making Sense of Different Types of Crises: A Study of the Swedish Media Coverage of the Terror Attacks against the United States and the 
U.S. Attacks in Afghanistan. The Harvard International Journal of Press/Politics, 8(4), $54-75$.

Oehmer, F. (2010). Aggressor or Victim? How the Antagonists of the Lebanon War 2006 are constructed in German Newspapers. Conflict \& communication online, 9(1).

Robinson, P., Goddard, P., Parry, K., \& Murray, C. (2009). Testing models of media performance in wartime: U.K. TV news and the 2003 invasion of Iraq. Journal of Communication, 59, 534-563.

Ruigrok, N., de Ridder, J. A., \& Scholten, O. (2005). News Coverage of the Bosnian War in Dutch Newspapers: Impact and Implications. In P. Seib (Hrsg.), Media and Conflict in the Twenty-First Century (S. 157-183). New York: Palgrave Macmillan.

Savarese, R. (1993). The European Press and Saladin the Fierce. European Journal of Coтmunication, 8, 53-75.

Scherer, H., Fröhlich, R., Scheufele, B., Dammert, S., \& Thomas, N. (2005). Bundeswehr, Bündnispolitik und Auslandseinsätze. Die Berichterstattung deutscher Qualitätszeitungen zur Sicherheits- und Verteidigungspolitik 1989 bis 2000. Medien \& Kommunikationswissenschaft, 53, 277-297.

Schreiber, W. (2008). Kriege und bewaffnete Konflikte 2008. Ein erster Überblick.

AKUF Analysen, Nr.2, Dezember 2008, http://www.sozialwiss.uni-hamburg.de/publish /Ipw/Akuf/publ/AKUF-Analysen-02.pdf. Zugegriffen: 10. Aug. 2009.

Shaw, D. L., \& Martin, S. E. (1993). The Natural, and Inevitable, Phases of War Reporting: Historical Shadows, New Communication in the Persian Gulf. In R. Denton (Hrsg.), The Media and the Persian Gulf War (S. 43-70). Westport: Praeger.

Shih, T-J., Wijaya, R., \& Brossard, D. (2008). Media Coverage of Public Health Epidemics: Linking Framing and Issue Attention Cycle Toward an Integrated Theory of Print News Coverage of Epidemics. Mass Communication \& Society. 11, 141-160.

Staiger, J. (2004). Selbstorganisation, Nicht-Linearität, Viabilität. Eine konstruktivistischsozialsystemische Perspektive auf Kriegsberichterstattung. In M. Löffelholz (Hrsg.), 
Krieg als Medienereignis II. Krisenkommunikation im 21. Jahrhundert (S. 145-168).

Wiesbaden: VS.

Vasterman, P. L. M. (2005). Media-Hype: Self-reinforcing news waves, journalistic standards and the construction of social problems. European Journal of Communication, 20, 508530

Vliegenthart, R., \& Walgrave, S. (2008). The Contingency of Intermedia Agenda Setting: A Longitudinal Study in Belgium. Journalism \& Mass Communication Quarterly, 85, $860-877$.

Waldherr, A. (2012). Die Dynamik der Medienaufmerksamkeit: Ein Simulationsmodell. BadenBaden: Nomos.

Weßler, H. (1999). Öffentlichkeit als Prozess. Deutungsstrukturen und Deutungswandel in der deutschen Drogenberichterstattung. Opladen: Westdeutscher.

Wien, C., \& Elmelund-Præstekær, C. (2009). An anatomy of media hypes: Developing a model for the dynamics and structure of intense media coverage of single issues. European Journal of Communication, 24, 183-201.

Wilke, J. (2005). Krieg als Medienereignis. Zur Geschichte seiner Vermittlung in der Neuzeit. In H.-P. Preußer (Hrsg.), Krieg in den Medien (S. 83-104). Amsterdam: Rodopi.

Wolfsfeld, G., \& Sheafer, T. (2006). Competing actors and the construction of political news:

The contest over waves in Israel. Political Communication, 23, 333-354.

Zelizer, B. (1992). CNN, the Gulf War, and Journalistic Practice. Journal of Communication, $42,66-81$.

Peter Miltner, Dipl. rer. com., ist wissenschaftlicher Mitarbeiter an der Arbeitsstelle Kommunikationstheorie/Medienwirkungsforschung des Instituts für Publizistik- und Kommunikationswissenschaft der Freien Universität Berlin.

Dr. Annie Waldherr ist wissenschaftliche Mitarbeiterin an der Arbeitsstelle Kommunikationstheorie/Medienwirkungsforschung des Instituts für Publizistik- und Kommunikationswissenschaft der Freien Universität Berlin. 